Document downloaded from:

http://hdl.handle.net/10251/160301

This paper must be cited as:

Erdogan, E.; Sánchez Pérez, EA.; Gok, O. (2019). Product factorability of integral bilinear operators on Banach function spaces. Positivity. 23(3):671-696.

https://doi.org/10.1007/s11117-018-0632-z

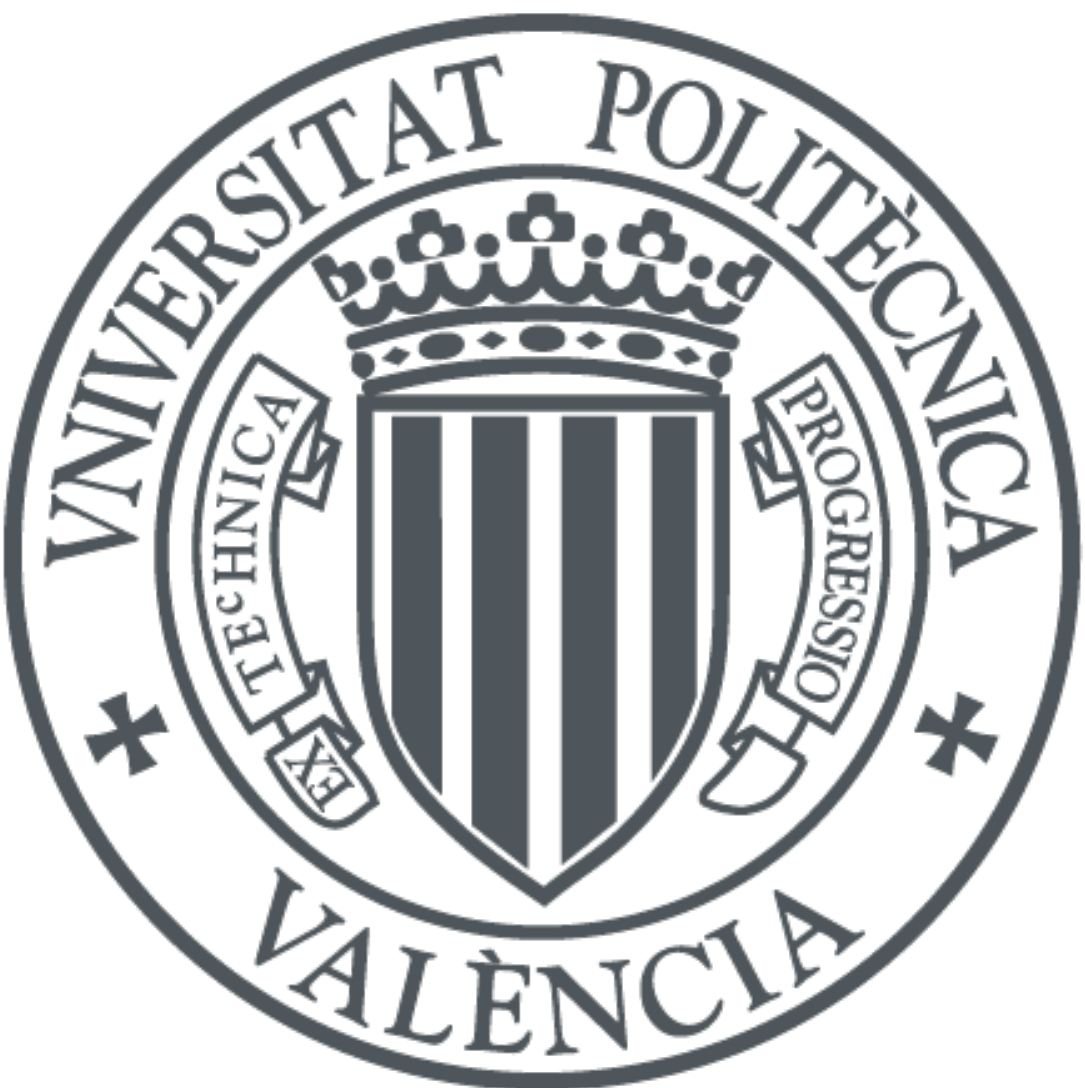

The final publication is available at

https://doi.org/10.1007/s11117-018-0632-z

Copyright Springer-Verlag

Additional Information 


\title{
PRODUCT FACTORABILITY OF INTEGRAL BILINEAR OPERATORS ON BANACH FUNCTION SPACES
}

\author{
E. ERDOĞAN AND E. A. SÁNCHEZ PÉREZ*
}

\begin{abstract}
This paper deals with bilinear operators acting in pairs of Banach function spaces that factor through the pointwise product. We find similar situations in different contexts of the functional analysis, including abstract vector lattices —orthosymmetric maps-, $C^{*}$-algebras —zero product preserving operators - , and classical and harmonic analysis -integral bilinear operators-. Bringing together the ideas of these areas, we show new factorization theorems and characterizations by means of norm inequalities. The objective of the paper is to apply these tools to provide new descriptions of some classes of bilinear integral operators, and to obtain integral representations for abstract classes of bilinear maps satisfying certain domination properties.
\end{abstract}

\section{INTRODUCTION}

A particular class of bilinear operators that play a fundamental role in Functional Analysis is the one defined by what we can call, broadly speaking, a product. We are thinking of for example, the internal product of a Banach algebra, but also on the pointwise product acting in a couple defined by a Banach function space and its Köthe dual as an $L^{1}$-valued bilinear map.

Consider a bilinear operator acting in a couple of Banach spaces in which a product is defined. If the bilinear map factors through such a product, some of the good properties of the factorization operator are preserved, and so we can use them for the analysis of the bilinear map. This general philosophy factorization of maps - is one of the main techniques that inspires classical and current developments in mathematical analysis. The first aim of the present paper is to develop the main results on factorization of bilinear maps acting in couples of Banach function spaces through the pointwise product $(\mu$-a.e). The second objective, to apply these results for the study of integral bilinear operators, a topic of current interest.

Concretely, in this paper we present a factorization theorem for bilinear continuous operators through a product map. It is given in terms of a summability condition, showing that this property also concerns zero product preserving bilinear maps. These are bilinear maps acting in a couple of Banach spaces in which a product is defined, and are zero valued in those couples of elements whose products are equal to zero. In a second step, we center our attention in the

2010 Mathematics Subject Classification. Primary: 47H60; Secondary: 46E30; 47A68; 47B38 .

Key words and phrases. bilinear operators; Banach function spaces; factorization; zero product preserving; symmetric operator.

The first author was supported by TÜBITAK- The Scientific and Technological Research Council of Turkey, Grant No:2211/E. The second author was supported by Ministerio de Ciencia, Innovación y Universidades, Agencia Estatal de Investigación (Spain) and FEDER, Grant MTM2016-77054-C2-1-P. 
case of bilinear maps acting in couples of Banach function spaces that are zero product preserving with respect to the $\mu$-a.e. pointwise product. We follow with an analysis of the main properties of these bilinear maps, including lattice type geometric properties and compactness. Finally, we adapt and apply our results to the case of some particular classes of integral bilinear operators.

Let us give now some information on the concept of zero product preserving bilinear operator. We must point out that this is a notion of transversal nature, affecting several branches of the theory of Banach spaces and the operator theory. We can find it — with different names - in several settings involving bilinear operators: for example, in the theory of Banach lattices, and in Fourier analysis. Classical operators acting in vector lattices that send disjoint elements to disjoint elements were introduced with the names disjointness preserving maps or separating maps; the notion was also carried to the framework of the function algebras (see $[1,8])$.

The notion of zero product preserving bilinear operator has been studied for bilinear maps $\phi: A \times A \rightarrow B$ acting in the Cartesian product of a Banach algebra $A ; \phi$ is zero product preserving if $a b=0$ implies $\phi(a, b)=0$ for $a, b \in$ $A$. In this context, we find that these maps are closely related with the so called separating maps. Indeed, for a separating map $S: A \rightarrow B$-that is a linear operator such that $S(a) S(b)=0$ if $a b=0$-, we can define the bilinear map $\phi(a, b)=S(a) S(b)$, which is clearly a zero product preserving bilinear map. These bilinear operators have been studied by some authors searching a good representation for them by means of linear operators. Due to the nice properties of the algebraic multiplication in a Banach algebra, a zero product preserving map can be factored through the product operator; we can find a lot of recently published papers regarding linear factorization of zero product preserving maps in the context of Banach algebras (see for example $[3,4,5,10,17,19]$ ). For instance, Alaminos et al showed that for some Banach algebras including the unital and $C^{*}$ - algebras, every zero product preseving bilinear operator can be factored through the algebraic multiplication composed with a linear map (see $[3,4,5])$.

On the other hand, in the context of abstract linear lattices Fremlin defined a new Archimedean vector lattice by means of a tensor product construction. In this context, the "product" of two Archimedean lattices is called the Archimedean vector lattice tensor product, or simply the Fremlin tensor product. Originally, he proved that every positive bilinear map can be uniquely factored through the tensor product of the involved spaces if the range space is a uniformly complete vector lattice [19]. Factorization for zero product preserving bilinear maps defined on vector lattices was considered by Buskes and van Rooij [10]. The term orthosymmetric is used for these operators, which in the case of Banach function spaces can be identified with the zero product preserving bilinear maps. Recall that in this case, two functions are disjoint if and only if their ( $\mu$-a.e.) pointwise product equals zero. An order bounded bilinear map $T: E \times E \rightarrow F$ is called orthosymmetric if $T(f, g)=0$ for the couples $f, g \in E$ such that $f \wedge g=0$ where $E$ and $F$ are the vector lattices, and they showed that if $E$ and $F$ are Archimedean then any orthosymmetric positive -that is, $T(x, y) \geq 0$ if $x \geq 0, y \geq 0$ - bilinear map satisfies the symmetry condition $T(x, y)=T(y, x)$. As a consequence of this theorem, it is seen that a bimorphism $B: E \times E \rightarrow F$ is symmetric if and only if it is orthosymmetric. The same authors gave the connection between the orthosymmetric bilinear operators and squares and powers of vector lattices (see 
[11]). Moreover, the commutators of orthosymmetric bilinear maps were recently investigated by Ben Amor, who gave a more general class of orthosymmetric bilinear maps related to the symmetry condition by defining the concept of relatively uniform continuity [6]. Finally, let us report that Erdoğan et al have given a factorization theorem for zero product preserving maps defined on the Cartesian product of Hilbert spaces through the convolution product [17]. For detailed information on the orthosymmetric operators we refer the interested reader to [9].

In the first part of the present paper we show a factorization theorem for Banach-space-valued bilinear operators $B: X \times Y \rightarrow Z$ acting in couples of Banach spaces through a particular product $\circledast: X \times Y \rightarrow G$. The first basic result of the paper states that such kind of factorization can be characterized by a summability condition associated to the product (Lemma 2.4). Our main technical theorem (Theorem 2.7) establishes that if we consider bilinear maps acting in Banach function spaces, and the pointwise product, this factorization gives the class of symmetric bilinear operators, that coincide with the so called zero product preserving operators - maps that are zero valued for couples of functions whose pointwise product is zero-- These results are presented in Section 2, in which we also provide some properties of this class of operators - more specific factorizations involving geometric requirements, Calderón spaces, compactness properties, among others - . In Section 3 we face the problem of enriching the factorization schemes for zero product preserving maps under some lattice geometric requirements expressed by domination inequalities. We will give a complete characterization of those bilinear maps that can be written as integrals with respect to a vector measure, or even as integrals with a Bochner density, by means of simple $q$-concavity-type inequality, obtaining in this way new representation formulas. Finally, in Section 4 we present an exhaustive analysis of what can be said when the definition of an integral bilinear operator involves the pointwise product composed with measurable transformations, starting with the generalized Hilbert bilinear transform as example. We show that this may provide a general new framework for understanding classical integral bilinear operators.

We will use standard notation and notions from Banach space theory. The letters $X, Y, Z$ describe the Banach spaces over the scalar field $\mathbb{K}=\mathbb{C}$ or $\mathbb{R}$ and $\mathbb{N}$ denotes the natural numbers. $B_{X}$ and $X^{*}$ denote the unit ball and the topological dual of a Banach space $X$, respectively. We write $\chi_{A}$ to denote the characteristic function of a given set $A$. The space of all bounded linear and bilinear operators will be denoted by $L(X, Y)$ and $B(X \times Y, Z)$, respectively.

Let $(\Omega, \Sigma, \mu)$ be a complete $\sigma$-finite measure space. $L^{0}(\mu)$ denotes the space of (equivalence classes of) all $\mu$-measurable functions on $\Omega . L^{p}(\mu)(p \geq 1)$ is the Banach space of functions for which the p-th power of the absolute value is $\mu$ integrable equipped with its standard norm $\|f\|=\left(\int_{\Omega}|f|^{p} d \mu\right)^{1 / p}$. For a positive real number $p, \ell^{p}(\Gamma)$ denotes the space of absolutely $p$-summable sequences.

A Banach space $(X(\mu),\|\cdot\|)$ of (equivalence classes of) $\mu$-measurable functions is a Banach function space - sometimes called also a Köthe function space(briefly B.f.s) over $\mu$ if

(i) if $g \in X(\mu)$ and $f$ is a measurable function such that $|f| \leq|g| \mu$-a.e., then $f \in X(\mu)$ and $\|f\| \leq\|g\|$,

(ii) for all $A \in \Sigma$ with positive measure there exists $B \in \Sigma$ such that $B \subset$ $A, \mu(B)>0$ and $\chi_{B} \in X(\mu)$. 
The assumption (ii) is equivalent to saturation property, that is, there is no $A \in \Sigma$ with $\mu(A)>0$ such that $f \chi_{A}=0$ a.e. for all $f \in X(\mu)$. Since the measure space is assumed to be $\sigma$-finite, this is also equivalent to $X(\mu)$ having a weak unit, i.e. a function $g \in X(\mu)$ such that $g>0$ a.e. (see [22]).

We will shortly write $X$ instead of $X(\mu)$ if the measure is clear in the context.

A Banach function space $X(\mu)$ is order continuous (briefly o.c.) if decreasing positive sequences converging $\mu$-a.e. to 0 converge also in the norm. It is known that the set of simple functions is dense in an order continuous Banach function space [22, Lemma 3]. The Köthe dual, also called associate space of a Banach function space $X$ is the Banach function space defined by

$$
X^{\prime}=\left\{f \in L^{0}(\mu): \int_{\Omega}|f g| d \mu<\infty \text { for all } g \in X\right\} .
$$

It coincides with the topological dual $X^{*}$ if and only if the Banach function space $X$ is order continuous (see [26, Section 1.b]). A Banach function space $X(\mu)$ has the Fatou property if any increasing positive sequence $\left\{f_{n}\right\}_{n}$ converging $\mu$-a.e. to a measurable function $f$ with $\sup _{n \in \mathbb{N}}\left\|f_{n}\right\|_{X(\mu)}<\infty$ implies $f \in X(\mu)$ and $\left\|f_{n}\right\|_{X(\mu)} \nearrow\|f\|_{X(\mu)}$. The Köthe dual $X^{\prime}$ of any Banach function space $X$ has the Fatou property.

We will write $E^{(p)}(p \geq 1)$ for the $p$-convexification of the Banach lattice $E$ in the sense of $[26$, Ch. 1.d] (see also the equivalent notion of $1 / p$-th power in $[29$, Ch.2] for a more explicit description). Recall that, when $E$ is a Banach function space, this is the space defined by the functions $f$ that satisfy $|f|^{p} \in E$. In this case, $E^{(p)}$ is also a Banach function space with the norm $\|f\|_{E^{(p)}}=\left\||f|^{p}\right\|_{E}^{1 / p}$, for $f \in E$ (see [28, Prop.1]).

For a couple of Banach function spaces $\left(E_{0}, E_{1}\right)$ and a concave, positively homogeneous function $\varphi:[0, \infty) \times[0, \infty) \rightarrow[0, \infty)$ such that $\varphi(a, b)=0 \Leftrightarrow$ $a=b=0$, the Calderón- Lozanovskii space $\varphi\left(E_{0}, E_{1}\right)$ generated by the couple $\left(E_{0}, E_{1}\right)$ and the function $\varphi$ is defined as all $z \in L^{0}(\Omega)$ such that for some $a \in E_{0}, b \in E_{1}$ with $\|a\|_{E_{0}} \leq 1,\|b\|_{E_{1}} \leq 1$ and for $\alpha>0$ we have $|z| \leq \alpha \varphi(|a|,|b|)$ a.e. on $\Omega$. The norm of an element $z \in \varphi\left(E_{0}, E_{1}\right)$ is the infimum of $\alpha$ satisfying the above inequality. If the power function $\varphi_{\theta}(a, b)=a^{\theta} b^{1-\theta}(0<\theta<1), \varphi_{\theta}\left(E_{0}, E_{1}\right)$ is the Calderón space $E_{0}^{\theta} E_{1}^{1-\theta}$ (see [13]).

A continuous linear operator between Banach spaces $T: X \rightarrow Y$ is said to be $(p, q)$-summing $\left(T \in \Pi_{p, q}(X, Y)\right)$ if there is a constant $k>0$ such that for every $x_{1}, \ldots, x_{n} \in X$ and for all positive integers $n$,

$$
\left(\sum_{i=1}^{n}\left\|T\left(x_{i}\right)\right\|_{Y}^{p}\right)^{1 / p} \leq k \sup _{x^{\prime} \in B_{X^{\prime}}}\left(\sum_{i=1}^{n}\left|\left\langle x_{i}, x^{\prime}\right\rangle\right|^{q}\right)^{1 / q} .
$$

We say that an operator $T: X \rightarrow Y$ is $p$ summing if it is $(p, p)$-summing.

\section{Product factorability of Bilinear maps}

Let us fix some basic concepts and terminology regarding what can be understood as the general setting for the factorization of bilinear maps through a particular bilinear operator $\circledast$ that we call a product. In this paper, this operator $\circledast$ will be fixed as acting as the pointwise product in a couple of Banach function spaces that will be required to be compatible, as we will explain later. However, the fundamental factorization is satisfied for any product having the following property. 
Let $X$ and $Y$ be Banach spaces. Consider a Banach space valued bilinear map $\circledast: X \times Y \rightarrow Z,(x, y) \rightsquigarrow \circledast(x, y)=x \circledast y$. We will call it a norming preserving product (n.p. product for short) if the inclusion $B_{Z} \subseteq \circledast\left(B_{X} \times B_{Y}\right)$ holds, and we have that

$$
\|\circledast(x, y)\|_{Z}=\inf \left\{\left\|x^{\prime}\right\|_{X}\left\|y^{\prime}\right\|_{Y}: x^{\prime} \in X, y^{\prime} \in Y, \circledast(x, y)=\circledast\left(x^{\prime}, y^{\prime}\right)\right\},
$$

for every $(x, y) \in X \times Y$.

Remark 2.1. The fact that the pointwise product $X \odot Y$ of Banach function spaces $X$ and $Y$ is an n.p. product is related to the Fatou property of the spaces involved. In the case that

$$
G=X \odot Y=\{f \cdot g: f \in X, g \in Y\}
$$

is a Banach function space with the norm

$$
\|h\|_{X \odot Y}=\inf \left\{\|f\|_{X}\|g\|_{Y}: h=f g, f \in X, g \in Y\right\},
$$

we have that the Fatou property of both $X$ and $Y$ implies the Fatou property of $G$ (see [23, Corollary 1] or [32, Theorem 2.3]). By Theorem 2.4 in [32], we have that for all $h \in G$ there are $f \in X$ and $g \in Y$ such that $h=f \cdot g$ and $\|h\|_{G}=\|f\|_{X}\|g\|_{Y}$, what means that $B_{G} \subseteq \odot\left(B_{X} \times B_{Y}\right)$.

(Notation: we will use the symbol $\odot$ for the pointwise product of Banach function spaces in those cases in which we want to remark its nature as n.p. product. In other case, we will simply use the usual notation $X \cdot Y$.)

Example 2.2. Let us give a canonical example. The pointwise product $\odot$ : $\ell^{p} \times \ell^{q} \rightarrow \ell^{r},\left(\left(x_{n}\right),\left(y_{n}\right)\right) \rightsquigarrow x_{n} \cdot y_{n}$, where $1 \leq p, q \leq \infty, 1 / p+1 / q=1 / r$, is an n.p. product (see [23, Example 1] and the references therein).

As in the example above, the pointwise product defines a norming preserving product in several couples of Banach spaces in which a "pointwise product" makes sense. For more examples of n.p. products, including the Lorentz and Cesaro function spaces, see the references [7, 12, 23, 32].

Now, we state our fundamental tools. Using the terminology coming from Banach algebras, we say that a bilinear map $B: X \times Y \rightarrow Z$ is zero product preserving with respect to the n.p. product $\circledast$ if

$$
x \circledast y=0 \quad \text { implies } \quad B(x, y)=0
$$

for all $(x, y) \in X \times Y$.

Definition 2.3. Let $X, Y, Z$ be Banach spaces. We will say that a bilinear continuous operator $B: X \times Y \rightarrow Z$ is $\circledast$-factorable through an n.p. product $\circledast: X \times Y \rightarrow G$ if it can be written as $B=T \circ \circledast$ for a linear bounded map $T: G \rightarrow Z$.

Lemma 2.4. Consider a bilinear continuous operator $B: X \times Y \rightarrow Z$. The map $B$ is $\circledast$-factorable through the n.p. product $\circledast$ if and only if there exists a constant $k>0$ such that for every finite set of vectors $\left(x_{i}\right)_{i=1}^{n} \in X$ and $\left(y_{i}\right)_{i=1}^{n} \in Y$, the following inequality holds;

$$
\left\|\sum_{i=1}^{n} B\left(x_{i}, y_{i}\right)\right\|_{Z} \leq k\left\|\sum_{i=1}^{n} x_{i} \circledast y_{i}\right\|_{G} .
$$


In this case, the following triangular diagram commutes;

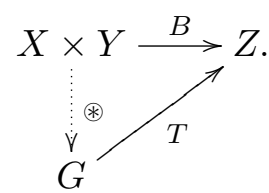

Proof. If the map $B$ is $\circledast$-factorable with the norming preserving product $\circledast$, then it can be factored through a linear continuous operator $T$ such that $B=T \circ \circledast$ by the definition of $\circledast$-factorability. Since $T$ is linear and continuous, it implies the required inequality as follows.

$$
\begin{aligned}
\left\|\sum_{i=1}^{n} B\left(x_{i}, y_{i}\right)\right\|_{Z} & =\left\|\sum_{i=1}^{n} T \circ \circledast\left(x_{i}, y_{i}\right)\right\|_{Z} \\
& =\left\|T\left(\sum_{i=1}^{n} x_{i} \circledast y_{i}\right)\right\|_{Z} \leq k\left\|\sum_{i=1}^{n} x_{i} \circledast y_{i}\right\|_{G} .
\end{aligned}
$$

For the converse, consider the map $T: X \circledast Y \rightarrow Z$ defined by

$$
T\left(\sum_{i=1}^{n} x_{i} \circledast y_{i}\right)=\sum_{i=1}^{n} B\left(x_{i}, y_{i}\right)=B^{\prime}\left(\sum_{i=1}^{n} x_{i} \otimes y_{i}\right)
$$

where $\sum_{i=1}^{n} x_{i} \otimes y_{i}$ is a tensor in the projective tensor product space $X \hat{\otimes}_{\pi} Y$ and $B^{\prime}$ denotes linearization of $B$ from $X \hat{\otimes}_{\pi} Y$ to $Z$ (see [30]).

The map $T$ is a well-defined and linear operator. Indeed, $T$ is linear since it is defined by the linear map $B^{\prime}$. To show that it is well-defined, consider $\sum_{i=1}^{n} x_{i} \circledast y_{i}=0$. Then by the inequality $(1)$, we get that $\left\|T\left(\sum_{i=1}^{n} x_{i} \circledast y_{i}\right)\right\|=$ $\left\|\sum_{i=1}^{n} B\left(x_{i}, y_{i}\right)\right\|_{Z}=0$. It follows that $T\left(\sum_{i=1}^{n} x_{i} \circledast y_{i}\right)=0$ and the map $T$ is well defined. Again from the inequality (1), it is easily seen that the linear map $T$ is bounded. This completes the proof.

From now on, we will center our attention in the case when the product $\circledast$ is the pointwise product among Banach function spaces $\odot$. Notice that this product is commutative and associative. Together with the specific structure of these spaces, this will allow to improve the basic characterization of $\circledast$-factorable operators given by Lemma 2.4 .

Let $X(\mu)$ and $Y(\mu)$ be Banach function spaces over the measure $\mu$. We will say that they are compatible - or that they form a compatible couple - if the product space

$$
X(\mu) \cdot Y(\mu):=\left\{f \cdot g \in L^{0}(\mu): f \in X(\mu), g \in Y(\mu)\right\}
$$

is a Banach function space again when it is endowed with the norm $\|h\|=$ $\inf \{\|f\|\|g\|: f \cdot g=h\}$, in case it is indeed a norm (see $[12,23,32]$ ).

Let us show now how Lemma 2.4 allows us to characterize the family of $\odot-$ factorable operators as the class of symmetric operators defined below. The reader can find - versions of - this definition in different articles. We follow the one given in [31].

Definition 2.5. Let $X(\mu), Y(\mu)$, and $Z(\mu)$ be Banach function spaces over the ( $\sigma$-finite) measure $\mu$. A continuous bilinear map $B: X(\mu) \times Y(\mu) \rightarrow Z(\mu)$ is called symmetric if the equality $B\left(\chi_{A}, \chi_{C}\right)=B\left(\chi_{A \cap C}, \chi_{A \cup C}\right)$ is satisfied for every $A, C \in \Sigma$. 
Remark 2.6. It is clearly seen that a symmetric bilinear map $B$ satisfies

$$
B\left(\chi_{A}, \chi_{C}\right)=B\left(\chi_{C}, \chi_{A}\right) \text { for every } A, C \in \Sigma,
$$

since

$$
B\left(\chi_{A}, \chi_{C}\right)=B\left(\chi_{A \cap C}, \chi_{A \cup C}\right)=B\left(\chi_{C \cap A}, \chi_{C \cup A}\right)=B\left(\chi_{C}, \chi_{A}\right) .
$$

The inverse is not true in general. To show that consider the bilinear continuous form $B: L^{1}(\mu) \times L^{1}(\mu) \rightarrow \mathbb{R}$ defined by $B(f, g)=\int f d \mu \cdot \int g d \mu$ for all $f, g \in L^{1}(\mu)$. It holds the equality $B\left(\chi_{A}, \chi_{C}\right)=B\left(\chi_{C}, \chi_{A}\right)$. Indeed, $B\left(\chi_{A}, \chi_{C}\right)=\mu(A) \cdot \mu(C)=$ $\mu(C) \cdot \mu(A)=B\left(\chi_{C}, \chi_{A}\right)$ for all $A, C \in \Sigma$.

However, it does not satisfy the equality $B\left(\chi_{A}, \chi_{C}\right)=B\left(\chi_{A \cap C}, \chi_{A \cup C}\right)$, since in general $B\left(\chi_{A}, \chi_{C}\right)=\mu(A) \cdot \mu(C) \neq \mu(A \cap C) \cdot \mu(A \cup C)=B\left(\chi_{A \cap C}, \chi_{A \cup C}\right)$. As a result, this shows that Definition 2.5 is not equivalent to the usual symmetry condition $B(f, g)=B(g, f)$ for all $f, g \in X$, where $B: X \times X \rightarrow Z$ is a bilinear continuous operator.

Theorem 2.7. Let $(\Omega, \Sigma, \mu)$ be a $\sigma$-finite measure space and let $X(\mu), Y(\mu)$ be B.f.s. over $\mu$ such that simple functions are dense in both $X(\mu)$ and $Y(\mu)$. Let $B$ be a continuous bilinear map $X(\mu) \times Y(\mu) \rightarrow E$, where $E$ is a Banach space. Suppose that there is a Banach function space $G(\mu)$ such that the pointwise product $\odot: X(\mu) \times Y(\mu) \rightarrow G(\mu)$ is an n.p. product. Then the following assertions are equivalent.

(1) $B$ is a symmetric operator.

(2) $B$ is $\odot$-factorable, that is, there is a continuous linear operator $R$ : $G(\mu) \rightarrow E$ such that $B=R \circ \odot$.

(3) There is $K>0$ such that for all $f_{1}, \ldots, f_{n} \in X(\mu)$ and $g_{1}, \ldots, g_{n} \in Y(\mu)$

$$
\left\|\sum_{i=1}^{n} B\left(f_{i}, g_{i}\right)\right\|_{E} \leq K\left\|\sum_{i=1}^{n} f_{i} \odot g_{i}\right\|_{G(\mu)} .
$$

(4) The operator $B$ is zero product preserving. That is, $B(f, g)=0$ whenever $f \odot g=0$ for all $f \in X(\mu)$ and $g \in Y(\mu)$.

Proof. Let us assume that $B: X(\mu) \times Y(\mu) \rightarrow E$ is a symmetric operator. Thus, we get $B\left(\chi_{A}, \chi_{C}\right)=0$ whenever $\mu(A \cap C)=0$.

Since $\Omega$ is $\sigma$-finite, then there exists a sequence $\left\{E_{k}\right\}_{k \in \mathbb{N}}$ in $\Sigma$ such that $\Omega=$ $\bigcup_{k=1}^{\infty} E_{k}$ and $\mu\left(E_{k}\right)<\infty$ for all $k \in \mathbb{N}$. Let us define the sequence of increasing sets $Y_{m}=\bigcup_{k=1}^{m} E_{k}$. Consider a couple of simple functions $f=\sum_{i=1}^{p} \lambda_{i} \chi_{A_{i}}$ and $g=\sum_{j=1}^{r} \gamma_{j} \chi_{C_{j}}$, where $\left(A_{i}\right)$ and $\left(C_{j}\right)$ are sequences of pairwise disjoint measurable sets. Definition of the simple functions gives rise to define a common partition for each couple $(f, g)$ of simple functions. Let us rewrite them by a common partition $f=\sum_{i=1}^{r} \lambda_{i} \chi_{D_{i}}$ and $g=\sum_{j=1}^{r} \gamma_{j} \chi_{D_{j}}$, where $\left(D_{i}\right)$ is sequence of pairwise disjoint measurable sets.

By the properties of a characteristic function, the pointwise product of a simple function $f$ and $\chi_{Y_{m}}$ is obtained as $f \odot \chi_{Y_{m}}=\sum_{i=1}^{r} \lambda_{i} \chi_{D_{i}} \odot \chi_{Y_{m}}=\sum_{i=1}^{r} \lambda_{i}\left(\chi_{D_{i}} \odot\right.$ $\left.\chi_{Y_{m}}\right)=\sum_{i=1}^{r} \lambda_{i} \chi_{D_{i} \cap Y_{m}}$. For every $m \in \mathbb{N}$, let us define the bilinear operator $B_{m}: X(\mu) \times Y(\mu) \rightarrow E, B_{m}(f, g)=B\left(f \odot \chi_{Y_{m}}, g \odot \chi_{Y_{m}}\right)$. Then $\left\{B_{m}\right\}_{m \in \mathbb{N}}$ is a sequence of well-defined, continuous, bilinear maps. The symmetry and bilinearity properties of the operator $B$ gives the following equality with some set 
operations;

$$
\begin{aligned}
B_{m}(f, g) & =B\left(f \odot \chi_{Y_{m}}, g \odot \chi_{Y_{m}}\right)=B\left(\sum_{i=1}^{r} \lambda_{i} \chi_{D_{i} \cap Y_{m}}, \sum_{j=1}^{r} \gamma_{j} \chi_{D_{j} \cap Y_{m}}\right) \\
& =\sum_{i=1}^{r} \lambda_{i} \gamma_{i} B\left(\chi_{D_{i} \cap Y_{m}}, \chi_{D_{i} \cap Y_{m}}\right)=\sum_{i=1}^{r} \lambda_{i} \gamma_{i}\left[B\left(\chi_{D_{i} \cap Y_{m}}, \chi_{Y_{m}}\right)\right] \\
& =B\left(\sum_{i=1}^{r} \lambda_{i} \gamma_{i} \chi_{D_{i} \cap Y_{m}}, \chi_{Y_{m}}\right)=B\left(f \odot g \odot \chi_{Y_{m}}, \chi_{Y_{m}}\right) .
\end{aligned}
$$

Thus, $B_{m}(f, g)=B\left(f \odot g \odot \chi_{Y_{m}}, \chi_{Y_{m}}\right)$ holds for every couple of simple functions.

Now let us show $B_{m}(f, g)=B\left(f \odot g \odot \chi_{Y_{m}}, \chi_{Y_{m}}\right)$ holds for the elements of $X(\mu)$ and $Y(\mu)$ which are not simple functions. By density, there are sequences $\left\{f_{n}\right\}$ and $\left\{g_{n}\right\}$ of simple functions such that $f=\lim _{n \rightarrow \infty} f_{n}$ and $g=\lim _{n \rightarrow \infty} g_{n}$. Applying the separate continuity of both the bilinear map B and the product $\odot$, we obtain

$$
\begin{aligned}
B_{m}(f, g) & =\lim _{n \rightarrow \infty} B\left(f_{n} \odot \chi_{Y_{m}}, g_{n} \odot \chi_{Y_{m}}\right) \\
& =\lim _{n \rightarrow \infty} B\left(f_{n} \odot g_{n} \odot \chi_{Y_{m}}, \chi_{Y_{m}}\right) \\
& =B\left(\lim _{n \rightarrow \infty} f_{n} \odot \lim _{n \rightarrow \infty} g_{n} \odot \chi_{Y_{m}}, \chi_{Y_{m}}\right) \\
& =B\left(f \odot g \odot \chi_{Y_{m}}, \chi_{Y_{m}}\right)
\end{aligned}
$$

holds. Therefore, we get that for every $m \in \mathbb{N}$ the bilinear operator $B_{m}$ can be written as $B_{m}(f, g)=B\left(f \odot g \odot \chi_{Y_{m}}, \chi_{Y_{m}}\right)$ for all $f \in X(\mu), g \in Y(\mu)$.

Now define the map $R_{m}: G(\mu) \rightarrow E$ by $R_{m}(h)=B_{m}(f, g)=B(f \odot g \odot$ $\left.\chi_{Y_{m}}, \chi_{Y_{m}}\right)$ for every function $h=f \odot g$ and every $m \in \mathbb{N} .\left\{R_{m}\right\}_{m \in \mathbb{N}}$ is a sequence of well-defined continuous linear operators from $G(\mu)=X(\mu) \odot Y(\mu)$ to $E$. Since it is easy to see that it is well-defined and linear, we only show the continuity. By the continuity of $B$, and taking into account that $\odot$ is an n.p. product, we have that

$$
\sup _{h \in B_{G(\mu)}}\left\|R_{m}(h)\right\|_{E}=\sup _{(f, g) \in B_{X(\mu)} \times B_{Y(\mu)}}\left\|B\left(f \odot g \odot \chi_{Y_{m}}, \chi_{Y_{m}}\right)\right\|_{E}<\infty .
$$

Indeed, note that the supremun over all the pairs $(f, g)$, where the functions $f$ and $g$ are in the corresponding unit balls, coincides with the supremum for all functions $h$ in the ball of $G(\mu)$ as a direct consequence of the definition of n.p. product.

It follows that $\left\{R_{m}\right\}_{m \in \mathbb{N}}$ is a sequence of bounded, linear operators. Moreover, it is pointwise convergent. Indeed, for each $f \odot g$, the sequence $\left\{R_{m}(f \odot g)\right\}_{m \in \mathbb{N}}$ satisfies the following

$$
\begin{aligned}
\lim _{m \rightarrow \infty} R_{m}(f \odot g) & =\lim _{m \rightarrow \infty} B\left(f \odot \chi_{Y_{m}}, g \odot \chi_{Y_{m}}\right) \\
& =B\left(\lim _{m \rightarrow \infty} f \odot \chi_{Y_{m}}, \lim _{m \rightarrow \infty} g \odot \chi_{Y_{m}}\right) \\
& =B(f, g) .
\end{aligned}
$$

Consequently, we have the pointwise limit operator $R:=\lim _{m \rightarrow \infty} R_{m}$. It is clear that this operator is well-defined and linear. By the Uniform Boundedness theorem, it is obtained that $R$ is a continuous operator. This shows that there is a linear continuous operator $R: G(\mu) \rightarrow E$ such that $R(f \odot g)=B(f, g)$ for all $f \in X(\mu), g \in Y(\mu)$. 
Moreover, it is independent of the representation of $f \odot g$. Assume that $h=$ $f_{1} \odot g_{1}=f_{2} \odot g_{2}$. Then, $R\left(f_{1} \odot g_{1}\right)-R\left(f_{2} \odot g_{2}\right)=B\left(f_{1}, g_{1}\right)-B\left(f_{2}, g_{2}\right)=0$. Therefore, we obtain the required factorization for a symmetric operator. The equivalence of (2) and (3) is proved in Lemma 2.4, and it is obvious that (3) implies (4).

Finally, it only remains to show that for every $A, C \in \Sigma$, the symmetry condition $B\left(\chi_{A}, \chi_{C}\right)=B\left(\chi_{A \cap C}, \chi_{A \cup C}\right)$ holds when the operator is zero product preserving. The characteristic functions corresponding to the sets $A, C \in \Sigma$ satisfy $\chi_{A} \odot \chi_{C}=\chi_{A \cap C}$. It follows that $\chi_{A} \odot \chi_{C}=0 \mu$-a.e. if and only if $\mu(A \cap C)=0$. By considering the assumption (4), we conclude that $B\left(\chi_{A}, \chi_{C}\right)=0$ whenever $\mu(A \cap C)=0$. It is already trivial that $B\left(\chi_{A \cap C}, \chi_{A \cup C}\right)=0$ if $\mu(A \cap C)=0$. Thus, we get that the symmetry condition holds for disjoint sets. To see that it is satisfied for arbitrary sets, consider $M, N \in \Sigma$ such that $\mu(M \cap N) \neq 0$. By the fact that $B\left(\chi_{A}, \chi_{C}\right)=0$ whenever $\mu(A \cap C)=0$, the following equality holds for the sets $M, N \in \Sigma$ by set operations and properties of the characteristic functions;

$$
\begin{aligned}
B\left(\chi_{M}, \chi_{N}\right) & =B\left(\chi_{\left(M \cap N^{c}\right) \cup(M \cap N)}, \chi_{N}\right) \\
& =B\left(\chi_{\left(M \cap N^{c}\right)}+\chi_{(M \cap N)}-\chi_{\left(M \cap N^{c}\right) \cap(M \cap N)}, \chi_{N}\right) \\
& =B\left(\chi_{(M \cap N)}, \chi_{N}\right)=B\left(\chi_{(M \cap N)}, \chi_{\left(N \cap M^{c}\right) \cup(N \cap M)}\right) \\
& =B\left(\chi_{(M \cap N)}, \chi_{(N \cap M)}\right)=B\left(\chi_{(M \cap N)}, \chi_{(N \cup M)}\right)
\end{aligned}
$$

Thus, the equality $B\left(\chi_{M}, \chi_{N}\right)=B\left(\chi_{(M \cap N)}, \chi_{(M \cup N)}\right)$ is obtained for arbitrary sets $M, N \in \Sigma$ and it follows that $B$ is a symmetric operator. This completes the proof.

Remark 2.8. If we have a finite measure space $(\Omega, \Sigma, \mu)$, the factorization is obtained easily. Indeed, $\chi_{\Omega} \in X(\mu)$ and $\chi_{\Omega} \in Y(\mu)$ since the measure $\mu$ is finite. We can obtain in this case an equivalent definition of symmetric operators, that is, the map $B$ is symmetric if and only if $B\left(\chi_{A}, \chi_{C}\right)=B\left(\chi_{A \cap C}, \chi_{\Omega}\right)$ for all $A, C \in \Sigma$. Using the density as in the proof of the Theorem 2.7, we get that for a symmetric operator holds the equality $B(f, g)=B\left(f g, \chi_{\Omega}\right)$ for all $f \in X(\mu), g \in$ $Y(\mu)$. If we define a map $T: G(\mu) \rightarrow E, T(h)=T(f \odot g)=B\left(f \odot g, \chi_{\Omega}\right)$, we get a bounded linear continuous operator $T$ such that $B:=T \circ \odot$.

Remark 2.9. The abstract requirement on the space $G(\mu)$ in Theorem 2.7 is clearly fulfilled when $G(\mu)$ is the product space of $X(\mu)$ and $Y(\mu)$, when they form a compatible couple. This can be easily checked just by considering its definition. Order continuity of this space will be relevant through the paper, in particular because it implies density of simple functions. When a product space is order continuous in terms of the properties of the factor spaces is nowadays well-known. The reader can find complete characterizations or sufficient conditions for this property to hold in several recent papers. For example, the reader can find in Section 5 of [15] (Corollary 5.3) the following result: if $X(\mu)$ and $Y(\mu)$ define a compatible couple of order continuous B.f.s. over a finite measure $\mu$ such that $X(\mu) \subseteq Y(\mu)^{\prime}$, then the product $X(\mu) \cdot Y(\mu)$ is order continuous. Another result in this direction is the following. It is known that $X(\mu) \cdot Y(\mu)=\left(X(\mu)^{1 / 2} Y(\mu)^{1 / 2}\right)^{(1 / 2)}$ for the $B a$ nach function spaces $X(\mu)$ and $Y(\mu)$, where $X(\mu)^{1 / 2} Y(\mu)^{1 / 2}$ is the interpolation lattice, also called the Calderón space [23, Theorem 1(iv)]. Then $X(\mu) \cdot Y(\mu)$ is order continuous if at least one of the spaces $X(\mu)$ and $Y(\mu)$ is order continuous [32]. 
A necessary and sufficient condition for the order continuity of the Banach function space $X(\mu) \cdot Y(\mu)$ can be given by means of the notion of jointly order discontinuity. Let $X_{a}$ denote the subspace of all order continuous elements of the Banach function space $X$, that is, the space of the elements $f \in X$ such that for any sequence $\left(f_{n}\right) \subset X$ satisfying $0 \leq f_{n} \leq|f|$ and $f_{n} \rightarrow 0 \mu$-a.e. one has $\|f\|_{X} \rightarrow 0$. A couple of Köthe spaces $(X, Y)$ is said to be jointly order discontinuous if there are elements $f \in X \backslash X_{a}, g \in Y \backslash Y_{a}$ and a sequence of measurable sets $A_{n} \searrow \emptyset$ such that for each sequence $\left(B_{n}\right) \in \Sigma$ with $B_{n} \subset A_{n}$ for all $n \in \mathbb{N}$ there are a number $a>0$ and a subsequence $\left(n_{k}\right) \in \mathbb{N}$ such that either

$$
\left\|f \chi_{B_{n_{k}}}\right\|_{X} \geq a \text { and }\left\|g \chi_{B_{n_{k}}}\right\|_{Y} \geq a \text { for all } k \in \mathbb{N},
$$

or

$$
\left\|f \chi_{B_{n_{k}}^{\prime}}\right\|_{X} \geq a \text { and }\left\|g \chi_{B_{n_{k}}^{\prime}}\right\|_{Y} \geq \text { a for all } k \in \mathbb{N},
$$

where $B_{n}^{\prime}=A_{n} \backslash B_{n}$ (see [24, Definition 12]). Corollary 1 in the paper [23] states that the Banach function space $X(\mu) \cdot Y(\mu)$ is order continuous if and only if $X(\mu)$ and $Y(\mu)$ are not jointly order discontinuous.

2.1. Factorization through the $r$-convexification of Banach function spaces. For the aim of simplicity, in this subsection and the next one we will use the notation $E$ for a Banach function space over a fixed measure $\mu$.

Remark 2.10. Let us consider the bilinear operator defined by the ( $\mu$-a.e.) pointwise product $\odot: E^{(p)} \times E^{(q)} \rightarrow E^{(r)},(f, g) \rightsquigarrow f \cdot g$, where $\frac{1}{p}+\frac{1}{q}=\frac{1}{r}$ for $1 \leq r<p, q<\infty$. This bilinear map is a norming preserving product (cf. [23, Example 1], [28, Lemma 1] or [29, Lemma 2.21(i)]).

Note that if we consider the Banach function space $E=L^{1}(\mu)$ we obtain that the pointwise product is an n.p. product from $L^{p}(\mu) \times L^{q}(\mu)$ to $L^{r}(\mu)$. It follows immediately by the definition that the $p$-convexification $E^{(p)}(0<p<\infty)$ of $E$ is order continuous, if $E$ is. Therefore, in this case simple functions are dense in $E^{(p)}, 1 \leq p<\infty$. We immediately obtain the following corollary.

Corollary 2.11. Let $E$ be an order continuous Banach function space. Let $1 \leq$ $r<p, q<\infty$, where $\frac{1}{p}+\frac{1}{q}=\frac{1}{r}$. If $B: E^{(p)} \times E^{(q)} \rightarrow Y$ is a Banach space valued bilinear continuous operator, the following statements imply each other.

(i) The operator $B$ is symmetric.

(ii) The bilinear operator $B$ is $\odot$-factorable, that is, there exists a bounded operator $T: E^{(r)} \rightarrow Y$ such that $B:=T \circ \odot$.

(iii) The operator $B$ is zero product preserving.

2.2. Factorization through the duality map acting in Banach function spaces. Let $E$ be an order continuous Banach function space over $\mu$ with the Fatou property and consider its Köthe dual space $E^{\prime}$. In this section we will show the case when we consider the pointwise product $\odot: E \times E^{\prime} \rightarrow L^{1}(\mu)$ associated to the duality map, as product. Several well-known results allow to assert that it is in fact an n.p. product.

Remark 2.12. Recall that the well-known factorization theorem of Lozanovskii states that for any Banach function space $E$ having the Fatou property and its associate space $E^{\prime}$, the product space $E \odot E^{\prime}:=E \cdot E^{\prime}$ is a product Banach function space that is isometrically equal to $L^{1}(\mu)$ (see [27], also [20]). In other words, E and $E^{\prime}$ always form a compatible couple. 
By Theorem 2.7 we immediately obtain the following.

Corollary 2.13. Let the set of simple functions be dense in both $E$ and its associate space $E^{\prime}$, and assume that $E$ has the Fatou property and $Y$ is a Banach space. Then, for any bilinear continuous operator $B: E \times E^{\prime} \rightarrow Y$ the following statements are equivalent.

(i) The bilinear operator $B: E \times E^{\prime} \rightarrow Y$ is $\odot$-factorable.

(ii) The operator $B$ is zero product preserving, that is, for each pair of elements $f \in E$ and $h \in E^{\prime}$ we have that

$$
\langle f, h\rangle=\int_{\Omega} f h d \mu=0 \quad \Rightarrow \quad B(f, h)=0 .
$$

(iii) The operator $B$ is symmetric.

Unifying the classical setting for the relation among the Calderón construction and the pointwise product, Kolwicz et al have considered the product spaces with the Calderón construction in [23]. For example, for a couple of Banach function spaces $X(\mu)$ and $Y(\mu)$ the following isometric equalities hold: $X(\mu)^{(p)} \odot$ $Y(\mu)^{\left(p^{\prime}\right)}=X(\mu)^{1 / p} Y(\mu)^{1 / p^{\prime}}$ for $1<p<\infty$ and $\frac{1}{p}+\frac{1}{p^{\prime}}=1, X(\mu)^{(p)} \odot Y(\mu)^{(p)}=$ $(X(\mu) \odot Y(\mu))^{(p)}$ for $0<p<\infty$, and $X(\mu) \odot Y(\mu)=\left(X(\mu)^{1 / 2} Y(\mu)^{1 / 2}\right)^{(1 / 2)}$ (see $[23$, Theorem 1]). Thus, we get the following

Corollary 2.14. Let $X(\mu)$ and $Y(\mu)$ be order continuous Banach function spaces. Then

i) If $1<p<\infty$ and $\frac{1}{p}+\frac{1}{p^{\prime}}=1$, the Banach space valued symmetric bilinear continuous operator $B: X(\mu)^{(p)} \times Y(\mu)^{\left(p^{\prime}\right)} \rightarrow Z$ factors through a linear operator $T: X(\mu)^{1 / p} Y(\mu)^{1 / p^{\prime}} \rightarrow Z$, where $X(\mu)^{1 / p} Y(\mu)^{1 / p^{\prime}}$ is the corresponding Calderón space.

ii) If $X(\mu)^{(p)}$ and $Y(\mu)^{(p)}$ form a compatible couple, then every symmetric bilinear continuous operator $B: X(\mu)^{(p)} \times Y(\mu)^{(p)} \rightarrow Z$ factors through a linear operator $T:(X(\mu) \odot Y(\mu))^{(p)} \rightarrow Z$, where $0<p<\infty$.

iii) If $X(\mu)$ and $Y(\mu)$ form a compatible couple, then every symmetric bilinear continuous operator $B: X(\mu) \times Y(\mu) \rightarrow Z$ factors through a linear operator

$$
T:\left(X(\mu)^{1 / 2} Y(\mu)^{1 / 2}\right)^{(1 / 2)} \rightarrow Z .
$$

Corollary 2.15. Consider order continuous Banach function spaces $E, F$ and $G$ over the same measure space $\mu$. Suppose that $E$ and $F$ have the Fatou property, $G^{\prime}$ is order continuous, and $E \odot F=G$. Then if the bilinear continuous operator $B$ : $E \times F \rightarrow F$ is symmetric with factorization operator $T_{B}$, the bilinear continuous operator $A: E \times G^{\prime} \rightarrow G^{\prime}$ given by $A=T_{B}^{\prime} \circ \odot$ is symmetric too. Conversely, if a bilinear continuous operator $A: E \times G^{\prime} \rightarrow G^{\prime}$ is symmetric with factorization operator $T_{A}$, then the operator $B: E \times F \rightarrow F$ given by $B=T_{A}^{\prime} \circ \odot$ is also symmetric.

Proof. Assume that $B$ is a symmetric operator. Then there is a linear operator $T_{B}: G \rightarrow F$ defined by $B(e, f)=T(e \odot f), e \in E, f \in F$. The linear operator $T_{B}$ has an adjoint operator $T_{B}^{\prime}$ that can be defined having the image in $G^{\prime}$, due to the order continuity of $G$, and so $T_{B}^{\prime}: F^{\prime} \rightarrow G^{\prime}$ is defined by $\left\langle g, T_{B}^{\prime}\left(f^{\prime}\right)\right\rangle=\left\langle T_{B}(g), f^{\prime}\right\rangle$. Theorem 3.7 in [32] states that if $E, F$ have the Fatou property and $E \odot F=G$, then $E \odot G^{\prime}=F^{\prime}$ holds, and so $F^{\prime}$ is also a product Banach function space. Thus, we can write $f^{\prime}=e_{1} \odot g^{\prime}$ for every $f^{\prime} \in F^{\prime}$, where $e_{1} \in E$ and $g^{\prime} \in G^{\prime}$. 
It is clear that for the linear adjoint operator $T_{B}^{\prime}$ there is a symmetric operator $A: E \times G^{\prime} \rightarrow G^{\prime}$ defined by $T_{B}^{\prime}\left(f^{\prime}\right)=T_{B}^{\prime}\left(e_{1} \odot g^{\prime}\right)=A\left(e_{1}, g^{\prime}\right)$. Moreover by the definition of the adjoint operator we obtain the symmetric operator $A$ such that $A\left(e_{1}, g^{\prime}\right) g=\left\langle T_{B}(g), e_{1} \odot g^{\prime}\right\rangle$. Conversely, consider a symmetric operator $A: E \times G^{\prime} \rightarrow G^{\prime}$. Since the space $G^{\prime}$ is order continuous, it contains the simple functions densely. Thus, it allows us to get a factorization for the bilinear operator $A$ such that $T_{A}: F^{\prime} \rightarrow G^{\prime}$. Therefore, by using adequately the duality properties of the spaces $F, G$ we obtain a well-defined adjoint operator $T_{A}^{\prime}: G \rightarrow F$ and we conclude that the operator $B=T_{A}^{\prime} \circ \odot$ is also a symmetric operator.

Corollary 2.16. Suppose that the order continuous Banach function spaces $E, F, G$ defined over the same measure space have the Fatou property, and $E$ forms a compatible couple with both $F$ and $G$ such that $E \odot F=E \odot G$ isomorphically. Then, a bilinear continuous operator $B_{1}: E \times F \rightarrow Y$ is symmetric if and only if there is a symmetric bilinear continuous operator $B_{2}: E \times G \rightarrow Y$ and an isomorphism $\phi: F \rightarrow G$ such that $B_{2}(\cdot, \cdot)=B_{1}(\cdot, \phi(\cdot))$.

Proof. Let us assume that $B_{1}$ is symmetric, then it has a linear factorization $T_{1}$ : $E \odot F \rightarrow Y$ such that $B_{1}(e, g)=T_{1}(e \odot g)$. Since $E \odot F=E \odot G$ isomorphically, it follows that $F=G$ isomorphically (see [32, Corollary 2.6]). Therefore, we obtain a bilinear operator $B_{2}: E \times G \rightarrow Y$ defined by $B_{2}(e, g)=T_{1} \circ \odot \circ(I d \times \phi)(e, g)$, where $I d$ denotes the identity operator defined on $E$ and the $\phi$ is the isomorphism between the function spaces $F$ and $G$. Conversely, assume that $B_{2}$ is symmetric. Then, there is a linear operator $T_{2}: E \odot G \rightarrow Y$ such that $T_{2}(e \odot g)=B_{2}(e, g)$. Define the map $B_{1}(e, f)=T_{2} \circ \odot \circ\left(I d^{-1} \times \phi^{-1}\right)(e, f)=B_{2}(e, g)$. It is easily seen that this is a bilinear map and symmetric.

2.3. Compactness and weak compactness of symmetric operators. Let us show now some compactness properties of symmetric maps. Recall that we say that a continuous bilinear map is (weakly) compact if it maps the unit ball to a relatively (weakly) compact set. Let us consider an n.p. product defined from $X(\mu) \times Y(\mu)$ to $G(\mu)$ and a symmetric map $B: X(\mu) \times Y(\mu) \rightarrow Z$. Assume that simple functions are dense in both $X(\mu)$ and $Y(\mu)$. It is easily seen that the symmetric map $B$ is (weakly) compact if and only if the linear operator $T$ appearing in its factorization is (weakly) compact, due to the definition of the product.

Corollary 2.17. If $1<r<p, q<\infty$ and $\frac{1}{p}+\frac{1}{q}=\frac{1}{r}$, each symmetric bilinear continuous operator $B: L^{p}(\mu) \times L^{q}(\mu) \rightarrow Z$ is weakly compact.

Proof. By Corollary $2.11 B$ factors through a linear factorization operator $T$ : $L^{r}(\mu) \rightarrow Z$. Since $L^{r}(\mu)$ is reflexive for $1<r<\infty$, the linear operator $T$ is weakly compact. Therefore, the map $B$ is weakly compact.

Corollary 2.18. Let $X(\mu)$ and $Y(\mu)$ be an order continuous compatible couple with the Fatou property, and assume that $(X(\mu) \cdot Y(\mu))^{\prime}$ is order continuous. Then, any symmetric bilinear continuous operator $B: X(\mu) \times Y(\mu) \rightarrow Z$ is weakly compact.

Proof. Since both $X(\mu)$ and $Y(\mu)$ have order continous norm and Fatou property, the Banach function space $X(\mu) \cdot Y(\mu)$ has order continous norm and Fatou property, too (see [23, Corollary 1]). Then, direct dual spaces computations show that the assumption on the product $X(\mu) \cdot Y(\mu)$ implies that it is reflexive 
as a Banach space. By the symmetry of $B$, it factors through a reflexive space, and so it is weakly compact.

For a range space $Z$ with the Schur property this result can be improved. Recall that a Banach space has Schur property if weak and norm convergent sequences coincide in it: the sequence space $\ell^{1}$ has this property.

Corollary 2.19. Under the assumptions on the compatible couple defined by $X(\mu)$ and $Y(\mu)$ given in Corollary 2.18, we have that every symmetric bilinear continuous map $B: X(\mu) \times Y(\mu) \rightarrow Z$ is compact if $Z$ has the Schur property.

The following theorem is a consequence of some well-known results on integral representation of weakly compact linear operators defined on $L^{1}(\mu)$ and our previous arguments.

Theorem 2.20. Let $(\Omega, \Sigma, \mu)$ be a finite measure space. Assume that the set of simple functions is dense in both $E(\mu)$ and its associate space $E^{\prime}(\mu)$ and $E$ has the Fatou property. A symmetric bilinear continuous operator $B: E(\mu) \times E^{\prime}(\mu) \rightarrow Z$ is weakly compact if and only if it has a representation as $B(f, g)=\int_{\Omega} f g h d \mu$ for all $f \in E(\mu), g \in E^{\prime}(\mu)$, where $h$ is an essentially bounded $Z$-valued Bochner integrable function defined on $\mu$ with a $\mu$-essentially relatively weakly compact range.

Proof. The symmetric map $B$ has a linear factorization through $L^{1}(\mu)$, that is, there is an operator $T$ such that $B(f, g)=T(f \cdot g), T: L^{1}(\mu) \rightarrow Z$. On the other hand, $B$ is weakly compact if and only if $T$ is weakly compact by the definition of the product acting in B.f.s. Dunford-Pettis-Philips' theorem states that a linear operator $T$ defined on $L^{1}(\mu)$ to $Z$ is weakly compact if and only if there exists an essentially bounded $Z$-valued Bochner integrable function $h$ defined on $\mu$ with a $\mu$-essentially relatively weakly compact range such that $T(k)=\int_{\Omega} k h d \mu$ for all $k \in L^{1}(\mu)$ (see [18, Ch. III, Theorem 2.12]). Since $T(k)=T(f \cdot g)=B(f, g)$, we get $B(f, g)=\int_{\Omega} f g h d \mu$ for all $f \in E(\mu), g \in E^{\prime}(\mu)$. This gives the desired representation.

Recall now that a linear continuous operator $T$ between Banach lattices is called positive if $T(x) \geq 0$ whenever $x \geq 0$. Let us assume that the space $Z$ is also a Banach lattice. We will say that a bilinear operator $B: X(\mu) \times Y(\mu) \rightarrow Z$ is positive product preserving if $B(f, g) \geq 0$ whenever $f \odot g \geq 0$ for $f \in X(\mu)$ and $g \in Y(\mu)$. It is clear that a symmetric bilinear map $B: X(\mu) \times Y(\mu) \rightarrow Z$ with an order continuous compatible couple $X(\mu), Y(\mu)$ is positive product preserving if and only if its factorization operator $T: G(\mu) \rightarrow Z$ defined by $T(f \odot g)=B(f, g)$ is a positive linear operator.

Corollary 2.21. Let $X(\mu)$ and $Y(\mu)$ be a compatible couple such that both are order continuous. A symmetric positive product preserving bilinear map $B$ : $X(\mu) \times Y(\mu) \rightarrow \ell^{1}$ is weakly compact-hence, compact-if and only if the associate space $(X(\mu) \cdot Y(\mu))^{\prime}$ of $X(\mu) \cdot Y(\mu)$ has order continuous norm.

Proof. By Theorem 2.7, a symmetric map $B$ is weakly compact positive product preserving if and only if it has a weakly compact positive linear factorization operator $T: X(\mu) \cdot Y(\mu) \rightarrow \ell^{1}$ defined by $T(f \odot g)=B(f, g)$. It is known that a positive linear operator from a Banach function space to $\ell^{1}$ is weakly compact if and only if the associate space of its domain has order continuous norm (see [25, pp. 275]). Therefore, $T$ is weakly compact and compact by the Schur property 
of $\ell^{1}$ if and only if the associate space $(X(\mu) \cdot Y(\mu))^{\prime}$ has order continuous norm, what implies that $B$ is compact also.

For example, if $1<r<p, q<\infty$ and $\frac{1}{p}+\frac{1}{q}=\frac{1}{r}$, every symmetric positive product preserving bilinear map $B: L^{p}(\mu) \times L^{q}(\mu) \rightarrow \ell^{1}$ is compact. Finally, we show another result for $C(K)$-type spaces. Recall that a Banach lattice $Z$ has a strong order unit if there is an element $e$ in $Z$ with the property that for every $z \in Z$ there exists a real number $\alpha$ such that $|z| \leq \alpha e$.

Corollary 2.22. Let $Z$ be a Dedekind complete Banach lattice with a strong order unit. Consider a compatible couple $X(\mu)$ and $Y(\mu)$ such that both are order continuous. Then every symmetric bilinear continuous operator $B: X(\mu) \times Y(\mu) \rightarrow Z$ can be written as a difference of two positive product preserving symmetric bilinear continuous operators.

Proof. Since $B$ is symmetric, there is a linear operator acting in the factorization space $T: X(\mu) \cdot Y(\mu) \rightarrow Z$ such that $B$ factors through $T$. The operator $T$ is regular, that is, it can be written as a difference of two positive linear operators $T_{1}-T_{2}$, since the space $Z$ is Dedekind complete Banach lattice with a strong order unit (see $[2$, Theorem 4.1$]$ ). Thus, the operator $B$ can be written as $B=T \circ \odot=\left(T_{1}-T_{2}\right) \circ \odot=T_{1} \circ \odot-T_{2} \circ \odot$. Since $T_{1}$ and $T_{2}$ are positive linear operators, it follows that $T_{1} \circ \odot$ and $T_{2} \circ \odot$ are positive product preserving symmetric bilinear operators. Therefore, $B$ is written as a difference of two positive product preserving symmetric bilinear operators.

For finishing this section, let us provide some direct applications on summability of certain bilinear operators. Suppose that $E$ is a Banach function space over a measure $\mu$ with associate space $E^{\prime}$ and let $H$ be a Hilbert space. By Grothendieck's Theorem, we know that $L\left(L^{1}(\mu), H\right)=\Pi_{1}\left(L^{1}(\mu), H\right)[16$, Chapter 3]. Using this result and Pietsch Domination Theorem (see [16, Theorem $2.12]$ ), we directly obtain the next

Corollary 2.23. Let the set of simple functions be dense in both $E$ and $E^{\prime}$ and assume that $E$ has the Fatou property. For any symmetric Hilbert space valued bilinear continuous map $B: E \times E^{\prime} \rightarrow H$, there is a positive constant c such that the following equivalent statements hold.

i) For $f_{1}, f_{2}, \ldots, f_{n} \in E$ and $g_{1}, g_{2}, \ldots, g_{n} \in E^{\prime}$,

$$
\sum_{i=1}^{n}\left\|B\left(f_{i}, g_{i}\right)\right\|_{H} \leq c \sup _{\phi \in B_{L} \infty} \sum_{i=1}^{n}\left|\left\langle f_{i} \cdot g_{i}, \phi\right\rangle\right| .
$$

ii) For $f \in E$ and $g \in E^{\prime}$,

$$
\|B(f, g)\|_{H} \leq c \int_{\phi \in B_{L} \infty}|\langle f \cdot g, \phi\rangle| d \nu(\phi)
$$

where $\nu$ is regular probability measure on the unit ball of $L^{\infty}(\mu)$.

In particular by the Dunford-Pettis property of $L^{1}(\mu)$ the bilinear map factors through a completely continuous linear operator. 


\section{LATTICE GEOMETRIC INEQUALITIES AND INTEGRAL REPRESENTATIONS FOR} $\odot$-FACTORABLE MAPS

Recall that a Banach function space $X(\mu)$ is $p$-convex (with $p$-convexity constant equal to one), if for every $f_{1}, \ldots, f_{n} \in X(\mu)$,

$$
\left\|\left(\sum_{i=1}^{n}\left|f_{i}\right|^{p}\right)^{1 / p}\right\|_{X(\mu)} \leq\left(\sum_{i=1}^{n}\left\|f_{i}\right\|_{X(\mu)}^{p}\right)^{1 / p} .
$$

A Banach-space-valued linear operator $T: X(\mu) \rightarrow E$ is $p$-concave (with $p$ concavity constant equal to one) if for every $f_{1}, \ldots, f_{n} \in X(\mu)$,

$$
\left(\sum_{i=1}^{n}\left\|T\left(f_{i}\right)\right\|_{E}^{p}\right)^{1 / p} \leq\left\|\left(\sum_{i=1}^{n}\left|f_{i}\right|^{p}\right)^{1 / p}\right\|_{X(\mu)} .
$$

Let $\mu$ be a measure. As usual, we write $[f]_{\mu}$ for the equivalence class of almost everywhere equal measurable functions that are associated with $f$. Recall that, if $X(\mu)$ and $Z(\eta)$ are Banach function spaces such that $\eta \ll \mu$, if the identification $[f]_{\mu} \mapsto[f]_{\eta}$ is well-defined, we can use this assignation to define a (continuous) inclusion/quotient operator $X(\mu) \hookrightarrow Z(\eta)$.

We are going to apply some classical arguments on factorization of operators for giving a particular integral representation for linear maps with good concavity properties.

Theorem 3.1. Consider a compatible couple of Banach function spaces $X(\mu)$ and $Y(\mu)$ having order continuous norms. Suppose that the product space $X(\mu) \cdot Y(\mu)$ is p-convex for $1 \leq p<\infty$. Consider a bilinear (continuous) Banach-space-valued operator $B: X(\mu) \times Y(\mu) \rightarrow E$. The following statements are equivalent.

(i) For $f_{1}, \ldots, f_{n} \in X(\mu)$ and $g_{1}, \ldots, g_{n} \in Y(\mu)$,

$$
\left(\sum_{i=1}^{n}\left\|B\left(f_{i}, g_{i}\right)\right\|^{p}\right)^{1 / p} \leq\left\|\left(\sum_{i=1}^{n}\left|f_{i} \cdot g_{i}\right|^{p}\right)^{1 / p}\right\|_{X(\mu) \cdot Y(\mu)} .
$$

(ii) There are a multiplication operator $M_{h}: X(\mu) \cdot Y(\mu) \rightarrow L^{p}(\mu)$ such that $\left\|M_{h}\right\|=1$ and a linear operator $T: L^{p}(\mu) \rightarrow E$ such that $B$ factors as $B=T \circ M_{h} \circ \odot$, that is, it factors through the scheme

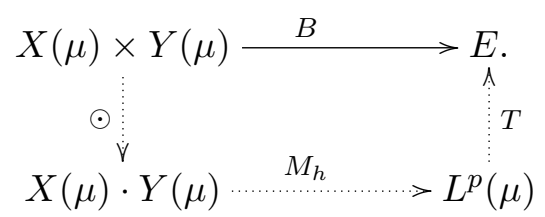

(iii) There is an E-valued vector measure $\nu$ such that $L^{p}(\mu) \hookrightarrow L^{1}(\nu)$, and

$$
B(f, g)=\int_{\Omega} f(t) g(t) h(t) d \nu(t)
$$

where $h$ defines a multiplication operator $M_{h}: X(\mu) \cdot Y(\mu) \rightarrow L^{p}(\mu)$.

Proof. (i) $\Rightarrow$ (ii) Note that the inequality in (i) directly implies that $B$ is 0 product preserving. This means by Theorem 2.7 that it factors through a linear continuous map $S: X(\mu) \cdot Y(\mu) \rightarrow E$ such that

$$
\left(\sum_{i=1}^{n}\left\|S\left(f_{i} \cdot g_{i}\right)\right\|^{p}\right)^{1 / p} \leq\left\|\left(\sum_{i=1}^{n}\left|f_{i} \cdot g_{i}\right|^{p}\right)^{1 / p}\right\|_{X(\mu) \cdot Y(\mu)}
$$


for $f_{1}, \ldots, f_{n} \in X(\mu)$ and $g_{1}, \ldots, g_{n} \in Y(\mu)$ by the inequality in (i) again. Consequently, we get that $S$ is $p$-concave. By hypothesis, we have that the product space $X(\mu) \cdot Y(\mu)$ is $p$-convex. A standard application of Maurey-Rosenthal argument $([14$, Cor. 5]) gives the existence of a norm one multiplication operator $M_{h}: X(\mu) \cdot Y(\mu) \rightarrow L^{p}(\mu)$ such that $S=T \circ M_{h}$, where $T: L^{p}(\mu) \rightarrow E$ is a linear continuous map. Composing all the elements, we get the desired diagram: $B=T \circ M_{h} \circ \odot$.

(ii) $\Rightarrow$ (iii) Since the space $L^{p}(\mu)$ is order continuous, we get that the operator $T: L^{p}(\mu) \rightarrow E$ defines a (countably additive) vector measure $\nu(A):=T\left(\chi_{A}\right)$, where $A \in \Sigma$. Moreover, we have that $L^{p}(\mu) \hookrightarrow L^{1}(\nu)$ due to the optimality of the space $L^{1}(\nu)$. The reader can find this result in [29, Th.4.14] under the assumption that $T$ is $\mu$-determined; note that by the proof given there, the result is still true if this is not the case, that is, if $T$ is not $\mu$-determined. It is wellknown that the space $L^{1}(\nu)$ is a Banach function space over a Rybakov measure $\eta$ for $\nu$, and $\eta \ll \mu$ because of the continuity of $T$; we can change then the inclusion by the identification of classes $[f]_{\mu} \mapsto[f]_{\eta}$, what is sometimes called an inclusion/quotient map, and the factorization is still preserved. Summing up all these comments, we get that

$$
B(f, g)=\int_{\Omega} f(t) g(t) h(t) d \nu(t)
$$

for all $f \in X(\mu)$ and $g \in Y(\mu)$.

(iii) $\Rightarrow$ (i) A direct computation just using the formula gives this implication. Indeed, if $f_{1}, \ldots, f_{n} \in X(\mu)$ and $g_{1}, \ldots, g_{n} \in Y(\mu)$,

$$
\begin{gathered}
\left(\sum_{i=1}^{n}\left\|B\left(f_{i}, g_{i}\right)\right\|_{E}^{p}\right)^{1 / p} \leq\left(\sum_{i=1}^{n}\left\|f_{i} g_{i} h\right\|_{L^{1}(\nu)}^{p}\right)^{1 / p} \leq\left(\sum_{i=1}^{n}\left\|f_{i} g_{i} h\right\|_{L^{p}(\mu)}^{p}\right)^{1 / p} \\
=\left\|h\left(\sum_{i=1}^{n}\left|f_{i} \cdot g_{i}\right|^{p}\right)^{1 / p}\right\|_{L^{p}(\mu)} \leq\left\|\left(\sum_{i=1}^{n}\left|f_{i} \cdot g_{i}\right|^{p}\right)^{1 / p}\right\|_{X(\mu) \cdot Y(\mu)}
\end{gathered}
$$

An integral with respect to a vector measure is still a rather abstract representation for the bilinear operator $B$. However, using the same result for $p=1$ we can still improve the representation for getting a kernel-type operator whenever the range space $E$ has the Radon-Nikodym property. Although we will show an special representation for the specific case of classical bilinear integral operators, we can improve the integral formula given above for the case of 0-product preserving bilinear operators factoring through a 1-concave linear map. As in Theorem 3.1, we suppose w.l.o.g. that the constant appearing in the inequality in (i) equals to 1 , that is, no specific constant appears.

Corollary 3.2. Let $\mu$ be a finite measure. Consider a compatible couple of Banach function spaces $X(\mu)$ and $Y(\mu)$ with order continuous norms. Suppose that $E$ is a Banach space with the Radon-Nikodym property. For a continuous bilinear operator $B: X(\mu) \times Y(\mu) \rightarrow E$, the following statements are equivalent.

(i) For $f_{1}, \ldots, f_{n} \in X(\mu)$ and $g_{1}, \ldots, g_{n} \in Y(\mu)$,

$$
\sum_{i=1}^{n}\left\|B\left(f_{i}, g_{i}\right)\right\| \leq\left\|\sum_{i=1}^{n}\left|f_{i} \cdot g_{i}\right|\right\|_{X(\mu) \cdot Y(\mu)} .
$$


(ii) There is a (norm one) E-valued vector measure Bochner integrable function $\Phi \in L^{\infty}(\mu, E)$ such that

$$
B(f, g)=\int_{\Omega} f(t) g(t) h(t) \Phi(t) d \mu(t),
$$

where $h \in(X(\mu) \cdot Y(\mu))^{\prime}$.

Proof. (i) $\Rightarrow$ (ii) Applying the assumption (i) and Theorem 3.1 we conclude that the variation of the vector measure is finite. Then, this result is obtained as an application of Theorem 3.1. Indeed, by hypothesis the vector measure $\nu$ provided by this theorem defines an operator $w \mapsto \int_{\Omega} w d \nu: L^{1}(\mu) \mapsto E$ that closes a factorization diagram as in the theorem. Using the Radon-Nikodym property of $E$ (see for instance [18] for a complete explanation of this notion) we get that there is an integrable vector-valued density $\Phi$ for the vector measure, in such a way that $d \nu=\Phi d \mu$ and so $w(t) \mapsto \int_{\Omega} \Phi(t) v(t) d \mu(t)$. This gives (ii).

(ii) $\Rightarrow$ (i) Taking into account that $\Sigma \ni A \mapsto \int_{A} \Phi d \nu \in E$ defines a vector measure, (iii) $\Rightarrow$ (i) in Theorem 3.1 for the case $p=1$ gives (i).

\section{Applichtions: WeAK REPRESENTAtion formulas FOR INTEGRAL BILINEAR OPERATORS}

In this section we apply the results given in the paper to some particular classes of bilinear operators that are defined by integral formulas. In order to do that, we will have to enlarge the notion of product preserving map by including some measurable transformations. We are interested in considering classical operators as the Hilbert transform, but the class we will deal with is broader than this. Let us start with a simple example.

Example 4.1. For $1 \leq p, q<\infty$ such that $\frac{1}{p}+\frac{1}{q}=\frac{1}{2}$, let us consider the bilinear operator $B: L^{p}([0,1]) \times L^{q}([0,1]) \rightarrow L^{2}([0,1])$ defined by

$$
B(f, g)(x)=\sum_{i=1}^{n}\left(\int_{0}^{1} r_{n}(y) f(y) g(y) d y\right) g_{n}(x),
$$

where $r_{n}$ denotes the $n^{\text {th }}$ Rademacher function,

$$
g_{n}(x)=2^{-(n+1) / 2} \chi_{\left[2^{-n}, 2^{(-n+1)}\right]}(x) \quad \text { for } x \in[0,1]
$$

and $n=1,2, \cdots$. It is clear that this bilinear operator is zero product preserving since $B(f, g)(x)=0$ if $f(y) g(y)=0$ a.e. for all $y \in[0,1]$. Therefore, by Theorem 2.7, it can be written as a linear integral operator such that $T(f \odot g)=T(h)=$ $\sum_{i=1}^{n}\left(\int_{0}^{1} r_{n}(y) h(y) d y\right) g_{n}(x)$, where $h \in L^{2}([0,1])=L^{p}([0,1]) \odot L^{q}([0,1])$.

Example 4.2. The Hilbert transform of a function $f(x)$ is given by

$$
H(f)(x)=\frac{1}{\pi} p \cdot v \cdot \int_{\mathbb{R}} f(x-t) \frac{d t}{t}
$$

where p.v. denotes the Cauchy principal value. This transform can be considered as the convolution of $f(x)$ with the tempered distribution $p \cdot v \cdot \frac{1}{\pi t}$. The bilinear Hilbert transform was introduced by Calderón as the following

$$
H_{\alpha_{1}, \alpha_{2}}(f, g)(x)=p . v \cdot \int_{\mathbb{R}} f\left(x-\alpha_{1} t\right) g\left(x-\alpha_{2} t\right) \frac{d t}{t} .
$$


Grafakos and $L i[21]$ have obtained a uniform bound for the bilinear Hilbert transform $H_{1, \alpha}: L^{p_{1}} \times L^{p_{2}} \rightarrow L^{p}$ for the real parameter $\alpha$ and $1>\frac{1}{p}=\frac{1}{p_{1}}+\frac{1}{p_{2}}>\frac{1}{2}$. If we suppose $\alpha=1$, then it is obviously seen that $H_{1,1}(f, g)(x)=(f \odot g) *\left(p \cdot v \cdot \frac{1}{t}\right)=$ $H(f \odot g)(x)$. Since $H_{1,1}(f, g)(x)=0$ if $f \odot g=0$, the operator $H_{1,1}$ is a zero product preserving map. Then it has a linear factorization that is the Hilbert transform defined on $L^{p_{1}} \odot L^{p_{2}}=L^{p}$ into $L^{p}$.

We can give a more general result for the bilinear Hilbert transform when it is considered as acting on products of Lorentz spaces. Villarroya defined the generalized bilinear Hilbert transform by using an arbitrary distribution instead of tempered distribution [33]. The generalized bilinear Hilbert transform is given by

$$
H_{u, \alpha}(f, g)(x)=\int_{\mathbb{R}} f(x-t) g(x-\alpha t) u(t) d t
$$

where $u$ is a distribution, $\alpha \in \mathbb{R}$ and $f, g$ are elements of the function space $C_{0}^{\infty}(\mathbb{R})$ of the smooth functions with a compact support in $\mathbb{R}$. A generalized bilinear Hilbert transform $H_{u, \alpha}$ is said to be $\left(p_{i}, q_{i}\right)_{i=1,2,3}$ bounded if it is possible to extend it to a bounded operator from $L^{p_{1}, q_{1}} \times L^{p_{2}, q_{2}}$ to $L^{p_{3}, q_{3}}$, where $0<p_{i}<$ $\infty, 0<q_{i}<\infty$ for $i=1,2,3$. Now, consider $a\left(p_{i}, q_{i}\right)_{i=1,2,3}$ bounded generalized Hilbert transform with the parameter $\alpha=1$ (where $L^{p_{i}, q_{i}}$ denotes the Lorentz function space, see [26, Section 2.a]). Then, it is seen that the transform $H_{u, 1}$ is a zero product preserving operator and $H_{u, 1}(f, g)(x)=(f \odot g) * u$. Since simple functions are dense in a Lorentz space $L^{p, q}$ for $0<p, q<\infty$, we get a factorization such that $T:\left[\left(L^{p_{1}, q_{1}}\right)^{1 / 2}\left(L^{p_{2}, q_{2}}\right)^{1 / 2}\right]^{(1 / 2)} \rightarrow L^{p_{3}, q_{3}}$ defined by $H_{u, 1}=$ $T(f \odot g)=(f \odot g) * u$, where $\left[\left(L^{p_{1}, q_{1}}\right)^{1 / 2}\left(L^{p_{2}, q_{2}}\right)^{1 / 2}\right]^{(1 / 2)}=L^{p_{1}, q_{1}} \odot L^{p_{2}, q_{2}}$.

Although pointwise product of functions appears explicitly in many of the classical examples of integral operators, most of them are not strictly 0-product preserving. For example, consider operators defined by the formula of the bilinear Hilbert transform given above but with compact support,

$$
H(f, g):=\int_{K} f(x-t) g(x-\alpha t) \frac{d t}{t}, \quad f, g \in L^{2}(\mu),
$$

where $(K, \Sigma, d t)$ is Lebesgue space on a compact set of the real line $K$, are not product preserving in general except that $\alpha=1$. In this section we show that is also possible to find a weak version of our representation theorem in this case. In order to do that, let us introduce some concepts and notation.

Let $(\Omega, \Sigma, \mu)$ be a $\sigma$-finite measure space and let $X(\mu)$ be a Banach function space over $\mu$. Let $\phi: \Omega \rightarrow \Omega$ a bimeasurable (measurable in both directions) bijection. We define $X_{\phi}(\mu)$ as the space of (classes of $\mu$-a.e. equal) functions

$$
X_{\phi}(\mu):=\left\{f \in L^{0}(\mu): f \circ \phi^{-1} \in X(\mu)\right\}
$$

endowed with the norm

$$
\|f\|_{X_{\phi}(\mu)}:=\left\|f\left(\phi^{-1}(\cdot)\right)\right\|_{X(\mu)}, \quad f \in X_{\phi}(\mu) .
$$

Note that such a $\phi$ defines an isometry, that is, the transformation

$$
\Delta_{\phi}: X(\mu) \rightarrow X_{\phi}(\mu) \text { given by } h \mapsto \Delta_{\phi}(h)=h \circ \phi \in X_{\phi}(\mu),
$$

that is clearly defined for all $h \in X(\mu)$, is an isometric isomorphism. The functions $\phi$ we are thinking about are typically simple transformations as, for the case of Lebesgue measure space $([0,1], \mathcal{B}([0,1]), d x), \phi_{1 / 2}(x)=x+1 / 2$ mod $1, x \in[0,1]$. If we take a rearrangement invariant space, for example if 
$X(\mu)=L^{p}([0,1])$ for $1 \leq p \leq \infty$, we have that $L_{\phi_{1 / 2}}^{p}([0,1])=L^{p}([0,1])$ isometrically.

We will consider couples of parametric families $\left\{\phi_{x}^{1}\right\}_{x \in \Omega}$ and $\left\{\phi_{x}^{2}\right\}_{x \in \Omega}$ of such bimeasurable bijections satisfying the requirement that $X_{\phi_{x}^{1}}(\mu)$ and $Y_{\phi_{x}^{2}}(\mu)$ are compatible for each $x \in \Omega$. Our idea is to recover using these tools a similar definition that the one that gives for example the bilinear Hilbert transform. Note that the simplest example of such a parametric family is when $\phi_{x}^{1}=\phi^{1}$ and $\phi_{x}^{2}=\phi^{2}$ for fixed functions $\phi^{1}$ and $\phi^{2}$; we use it just below.

We are now ready to define a general class of integral-type bilinear operators. Let $Z(\mu)$ be a Banach function space over $\mu$. Let $X(\mu)$ and $Y(\mu)$ be compatible Banach function spaces over $\mu$. In this context, we will say in what follows that a bilinear operator $B: X(\mu) \times Y(\mu) \rightarrow Z(\mu)$ is an integral bilinear operator if it is defined by a formula as

$$
B(f, g)(x):=\int_{\Omega} f\left(\phi_{x}^{1}(t)\right) g\left(\phi_{x}^{2}(t)\right) K(x, t) d t, \quad x \in \Omega, \quad f \in X(\mu), \quad g \in Y(\mu),
$$

where $K: \Omega \times \Omega \rightarrow \mathbb{R}$ is an integrable kernel such that the expression inside the integral is well-defined for each $x, t, f$ and $g$, and integrable, in such a way that $B(f, g)(\cdot) \in Z(\mu)$.

Independently of the case of pointwise type bounds depending on $x$ that we will explain later, we can get direct results when the functions $\phi^{1}$ and $\phi^{2}$ are fixed from the general framework constructed along the paper. So, let us assume for the next result that $\phi_{x}^{1}$ and $\phi_{x}^{2}$ do not depend on $x$. As a consequence of Lemma 2.4 we obtain the following general result. Note that it is not restricted to the case of integral bilinear operators, although it can be applied to this concrete context by its definition. It can be easily checked that the requirements in the following result are fulfilled in some simple — but meaningful - cases. For example, using for $\phi^{1}$ and $\phi^{2}$ the transformation $\phi_{1 / 2}$ explained above, we have that clearly the formula $f \circledast g:=f \circ \phi^{1} \cdot g \circ \phi^{2}$ defines an n.p. product.

Corollary 4.3. With the same notation and in the setting explained above, suppose that $X_{\phi^{1}}(\mu)$ and $Y_{\phi^{2}}(\mu)$ define a compatible couple. Assume also that the map given by $X(\mu) \times Y(\mu) \ni(f, g) \mapsto f \circ \phi^{1} \cdot g \circ \phi^{2}$, is an n.p. product. Then the following assertions are equivalent.

(i) There is a constant $k>0$ such that for every $f_{1}, \ldots, f_{n} \in X(\mu)$ and $g_{1}, \ldots, g_{n} \in Y(\mu)$

$$
\left\|\sum_{i=1}^{n} B\left(f_{i}, g_{i}\right)\right\| \leq k\left\|\sum_{i=1}^{n} f_{i} \circ \phi^{1} \cdot g_{i} \circ \phi^{2}\right\|_{X_{\phi^{1}}(\mu) \cdot Y_{\phi^{2}}(\mu)} .
$$

(ii) $B$ is an integral bilinear map that factors through $X_{\phi^{1}}(\mu) \cdot Y_{\phi^{2}}(\mu)$ as

$$
B(f, g)=T\left(f \circ \phi^{1} \cdot g \circ \phi^{2}\right), \quad f \in X(\mu), g \in Y(\mu),
$$

where $T: X_{\phi^{1}}(\mu) \cdot Y_{\phi^{2}}(\mu) \rightarrow Z(\mu)$ is a linear continuous operator.

Inspired in part by the example of the general Hilbert transform with compact support explained above, we start now to give a more accurate analysis of the problem of representing integral bilinear operators.

Lemma 4.4. Let $X(\mu)$ and $Y(\mu)$ be order continuous Banach function spaces over the measure space $(\Omega, \Sigma, \mu)$. Consider an integral bilinear operator $B$ : 
$X(\mu) \times Y(\mu) \rightarrow Z(\mu)$. Fix $x \in \Omega$, and let $\phi_{x}^{1}$ and $\phi_{x}^{2}$ be two measurable bijections defining isometries $X(\mu) \rightarrow X_{\phi_{x}^{1}}(\mu)$ and $Y(\mu) \rightarrow Y_{\phi_{x}^{2}}(\mu)$, respectively. Assume also that $X_{\phi_{x}^{1}}(\mu)$ and $Y_{\phi_{x}^{2}}(\mu)$ define a compatible couple.

Then there is a factorization through the product space $X_{\phi_{x}^{1}}(\mu) \cdot Y_{\phi_{x}^{2}}(\mu)$ of the bilinear continuous functional $B_{x}: X(\mu) \times Y(\mu) \rightarrow \mathbb{R}$ defined by

$$
B_{x}(f, g):=B(f, g)(x)=\int_{\Omega} f\left(\phi_{x}^{1}(t)\right) g\left(\phi_{x}^{2}(t)\right) K(x, t) d t, \quad f \in X(\mu), g \in Y(\mu) .
$$

Moreover, the functional $\varphi_{x} \in\left(X_{\phi_{x}^{1}}(\mu) \cdot Y_{\phi_{x}^{2}}(\mu)\right)^{*}$ that closes the factorization diagram is $\varphi_{x}(h(t)):=\int_{\Omega} h(t) K(x, t) d t \in \mathbb{R}$, and so we have that

$$
B(f, g)(x)=\left\langle f\left(\phi_{x}^{1}(\cdot)\right) \cdot g\left(\phi_{x}^{2}(\cdot)\right), \varphi_{x}(\cdot)\right\rangle, \quad f \in X(\mu), g \in Y(\mu) .
$$

Proof. It is worth noting that clearly order continuity of the spaces $X(\mu)$ and $Y(\mu)$ is automatically transferred to the spaces $X_{\phi_{x}^{1}}(\mu)$ and $Y_{\phi_{x}^{2}}(\mu)$. So, the lemma is just a consequence of the factorization theorem for zero product preserving operators and the construction. Indeed, taking into account that $\Delta_{\phi_{x}^{1}}$ and $\Delta_{\phi_{x}^{2}}$ are isometries, we can define a bilinear map

$$
B_{x}^{\prime}: X_{\phi_{x}^{1}}(\mu) \times Y_{\phi_{x}^{2}}(\mu) \rightarrow \mathbb{R}
$$

by $B_{x}^{\prime}=B_{x} \circ\left(\Delta_{\phi_{x}^{1}}^{-1} \times \Delta_{\phi_{x}^{2}}^{-1}\right)$. So, we have a factorization as

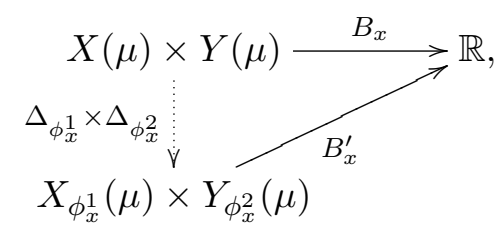

where $B_{x}^{\prime}$ is a bilinear integral and symmetric operator. Therefore, by Theorem 2.7, and taking into account that the spaces are order continuous - and so simple functions are dense-, it can be also factored as

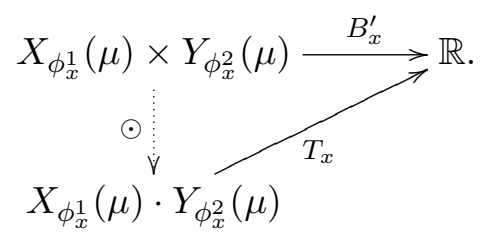

Once the existence of the factorization through the product space $X_{\phi_{x}^{1}}(\mu) \cdot Y_{\phi_{x}^{2}}(\mu)$ has been established, it is clear that $T_{x}$ has to be the linear and continuous functional

$$
\varphi_{x}(h):=h \mapsto \int_{\Omega} h(t) K(x, t) d t \in \mathbb{R},
$$

that belongs to the dual space $\left(X_{\phi_{x}^{1}}(\mu) \cdot Y_{\phi_{x}^{2}}(\mu)\right)^{*}$. Thus, we get that the pointwise evaluation of $B(f, g)$ at $x$ can be written as

$$
B_{x}(f, g)=B(f, g)(x)=\left\langle f\left(\phi_{x}^{1}(\cdot)\right) \cdot g\left(\phi_{x}^{2}(\cdot)\right), \varphi_{x}(\cdot)\right\rangle .
$$

Fubini's Theorem gives directly the next result.

Lemma 4.5. Let $Z(\mu)$ be order continuous. In the same setting and with the same notation that in Lemma 4.4 and assuming also that $K(x, t)$ (and so $\varphi_{x}$ ) 
depends only on $t$, - that is $\varphi_{x}=\varphi$ for all $x$ for a certain functional $\varphi-$, we have that for every element $\psi \in Z(\mu)^{\prime}$,

$$
\langle B(f, g), \psi\rangle=\left\langle\int_{\Omega}\left(f \circ \phi_{x}^{1}\right)(t) \cdot\left(g \circ \phi_{x}^{2}\right)(t) \psi(x) d \mu(x), \varphi(t)\right\rangle
$$

for every pair $f \in X(\mu)$ and $g \in Y(\mu)$.

The previous results suggest that our general class of integral bilinear operators can be analyzed in the product factorization framework constructed in the present paper whenever some requirements on the pointwise domination are assumed. Thus, next theorem gives a characterization of general bilinear operators by means of inequalities involving bimeasurable bijections. As the reader can see, the arguments used in the proof are adaptations of the ones we have used in the rest of the paper and belong to the same cycle of ideas. We use the notation of Lemmas 4.4 and 4.5. Note that, as we explained before, the requirement on the equality of the products is natural if we are working with the class of rearrangement invariant Banach function spaces.

Theorem 4.6. Let $X(\mu)$ and $Y(\mu)$ be a compatible couple of order continuous Banach function spaces such that its product is also order continuous, and consider a bilinear continuous operator $B: X(\mu) \times Y(\mu) \rightarrow Z(\mu)$. Consider a couple of parametric families $\left\{\phi_{x}^{1}\right\}_{x \in \Omega}$ and $\left\{\phi_{x}^{2}\right\}_{x \in \Omega}$ of bimeasurable bijections satisfying that $X_{\phi_{x}^{1}}(\mu) \cdot Y_{\phi_{x}^{2}}(\mu)=X(\mu) \cdot Y(\mu)$ isometrically for each $x \in \Omega$.

The following assertions are equivalent.

(i) There is a constant $k>0$ such that for every $f_{1}, \ldots, f_{n} \in X(\mu), g_{1}, \ldots, g_{n} \in$ $Y(\mu)$ and $x_{1}, \ldots, x_{n} \in \Omega$,

$$
\sum_{i=1}^{n} B\left(f_{i}, g_{i}\right)\left(x_{i}\right) \leq k\left\|\sum_{i=1}^{n} f_{i} \circ \phi_{x_{i}}^{1} \cdot g_{i} \circ \phi_{x_{i}}^{2}\right\|_{X(\mu) \cdot Y(\mu)} .
$$

(ii) There is a constant $k>0$ and a function $h_{0}$ such that the bilinear continuous map $B$ is an integral map that can be written as

$$
B(f, g)(x)=k \int_{\Omega}\left(f \circ \phi_{x}^{1}\right)(t) \cdot\left(g \circ \phi_{x}^{2}\right)(t) h_{0}(t) d \mu(t),
$$

where $f \in X(\mu), g \in Y(\mu), x \in \Omega$, and $h_{0} \in B_{(X(\mu) \cdot Y(\mu))^{\prime}}$.

Proof. For (i) $\Rightarrow$ (ii) we use a standard separation argument in a Maurey-Rosenthal fashion, as in the previous section. Consider all the functions $\Phi: B_{(X(\mu) \cdot Y(\mu))^{\prime}} \rightarrow$ $\mathbb{R}$ defined as

$$
\Phi(h):=\sum_{i=1}^{n} B\left(f_{i}, g_{i}\right)\left(x_{i}\right)-k \sum_{i=1}^{n} \int_{\Omega} f_{i} \circ \phi_{x_{i}}^{1} \cdot g_{i} \circ \phi_{x_{i}}^{2} h d \mu
$$

for given $f_{i} \in X(\mu), g_{i} \in Y(\mu)$ and $x_{i} \in \Omega$. Each of these functions is convex and weak* continuous, the whole family is concave and for each fixed function, by the Hahn-Banach Theorem and the inequality in (i), there is an element $h_{\Phi} \in B_{(X(\mu) \cdot Y(\mu))^{\prime}}$ such that $\Phi\left(h_{\Phi}\right) \leq 0$. Then we get a function $h_{0} \in B_{(X(\mu) \cdot Y(\mu))^{\prime}}$ such that

$$
B(f, g)(x) \leq k \int_{\Omega} f \circ \phi_{x}^{1} \cdot g \circ \phi_{x}^{2} h_{0}(t) d \mu(t)
$$

for all functions $f, g$ and all $x \in \Omega$. Since this must happen for all functions $f$ and $g$ we can change the signus in the inequality above just by changing for example 
$f$ by $-f$. Thus, we obtain for all $f, g$ and $x$ that

$$
B(f, g)(x)=k \int_{\Omega} f \circ \phi_{x}^{1} \cdot g \circ \phi_{x}^{2} h_{0}(t) d \mu(t) .
$$

(ii) $\Rightarrow$ (i) is given by a direct calculation.

The authors would like to thank the referee for the careful reading of the paper and some comments that helped to improve it.

\section{REFERENCES}

[1] Abramovich, Y.A., Kitover, A.K.: Inverses of disjointness preserving operators. Amer. Math. Soc. Providence, (2000)

[2] Abramovich, Y.A., Wickstead, A.W.: When each continuous operator is regular II. Indag. Math. (N.S.). 8(3), 281-294 (1997)

[3] Alaminos, J., Brešar, M., Extremera, J., Villena, A.R.: Maps preserving zero products. Studia Math. 193(2), 131-159 (2009)

[4] Alaminos, J., Brešar, M., Extremera, J., Villena, A.R.: On bilinear maps determined by rank one idempotents. Linear Algebra Appl. 432, 738-743 (2010)

[5] Alaminos, J., Extremera, J., Villena, A.R.: Orthogonality preserving linear maps on group algebras. Math. Proc. Camb. Phil. Soc. 158, 493-504 (2015)

[6] Ben Amor, F.: On orthosymmetric bilinear maps. Positivity. 14, 123-134 (2010)

[7] Astashkin, S.V., Maligranda, L.: Structure of Cesàro function spaces: a survey. Banach Center Publications. 102, 13-40 (2014)

[8] Beckenstein, E., Narici, L.: A non-Archimedean Stone-Banach theorem. Proc. Amer. Math. Soc. 100(2), 242-246 (1987)

[9] Bu, Q., Buskes, G., Kusraev, A.G.: Bilinear Maps on Products of Vector Lattices: A Survey, In: Boulabiar K., Buskes G., Triki A. (eds) Positivity. Trends in Mathematics, pp. 97-126 Springer, Birkhuser Basel (2007)

[10] Buskes, G., van Rooij, A.: Almost f-algebras: commutativity and Cauchy-Schwarz inequality. Positivity. 4, 227-231 (2000)

[11] Buskes, G., van Rooij, A.: Squares of Riesz spaces. Rocky Mountain J. Math. 31(1), 45-56 (2001)

[12] Calabuig, J.M., Delgado, O., Sánchez Pérez, E.A.: Generalized perfect spaces. Indag. Math. (N.S.). 19(3), 359-378 (2008)

[13] Calderón, A.P.: Intermediate spaces and interpolation, the complex method, Studia Math. 24, 113-190 (1964)

[14] Defant, A.: Variants of the Maurey-Rosenthal theorem for quasi Köthe function spaces. Positivity. 5, 153-175 (2001)

[15] Delgado Garrido, O., Sánchez Pérez, E. A.: Strong factorizations between couples of operators on Banach function spaces. Journal of Convex Analysis. 20(3), 599-616 (2013)

[16] Diestel, J., Jarchow, H., Tonge, A.: Absolutely Summing Operators, Vol. 43. Cambridge University Press (1995)

[17] Erdoğan, E., Calabuig, J.M., Sánchez Pérez, E.A.: Convolution-continuous bilinear operators acting in Hilbert spaces of integrable functions. Ann. Funct. Anal. 9(2), 166-179 (2018)

[18] Diestel, J., Uhl, J.J.: Vector Measures. Amer.Math. Soc, Providence (1977)

[19] Fremlin, D.H.: Tensor products of Archimedean vector lattices, Amer. J. Math. 94, 778-798 (1972)

[20] Gillespie, T.A.: Factorization in Banach function spaces. Nederl. Akad. Wetensch. Indag. Math. 43(3), 287-300 (1981)

[21] Grafakos, L., Li, X.: Uniform bounds for the bilinear Hilbert transforms I. Annals of Mathematics. 159, 889-933 (2004)

[22] Kantorovich, K.L., Akilov, G.P.: Functional Analysis, Nauka, Moscow 1977 (Russian); English transl. Pergamon Press, Oxford, Elmsford, New York (1982)

[23] Kolwicz, P., Leśnik, K., Maligranda, L.: Pointwise products of some Banach function spaces and factorization. J. Funct. Anal. 266(2), 616-659 (2014)

[24] Kolwicz, P., Leśnik, K.: Topological and geometrical structure of Calderón-Lozanovskii construction, Math. Inequal. Appl. 13(1), 175-196 (2010) 
[25] Kühn, B.: Banachverbände mit ordnungsstetiger dualnorm. Mathematische Zeitschrift. 167(3), 271-277 (1979)

[26] Lindenstrauss, J., Tzafriri, L.: Classical Banach spaces II: Function spaces. Vol. 97. Springer Verlag, Berlin Heidelberg, (1979)

[27] Lozanovskii, G. Ya.: On some Banach lattices. Sibirsk. Mat. Zh. 10, 584-599 (1969)(Russian); English transl. in Siberian Math. J. 10(3), 419-431 (1969)

[28] Maligranda, L., Persson, L.E.: Generalized duality of some Banach function spaces. Nederl. Akad. Wetensch. Indag. Math. 51(3), 323-338 (1989)

[29] Okada, S., Ricker, W., Sánchez Pérez, E.A.: Optimal domain and integral extension of operators. Oper. Theory Adv. Appl. Birkhäuser/Springer 180, (2008)

[30] Ryan, R.: Introduction to Tensor Product of Banach Spaces. Springer-Verlag, London (2002)

[31] Sánchez Pérez, E. A., Werner, D.: Slice continuity for operators and the Daugavet property for bilinear maps. Funct. Approx. Comment. Math. 50(2), 251-269 (2014)

[32] Schep, A.R.: Products and factors of Banach function spaces. Positivity. 14(2), 301-319 (2010)

[33] Villarroya, F.: Bilinear multipliers on Lorentz spaces. Czechoslovak Math. J. 58(4), 10451057 (2008)

E. ErdoĞan. Department of Mathematics, Faculty of Art and Science, UniverSity of Marmara, 34722, KadikÖy, Istanbul, Turkey.

E-mail address: ezgi.erdogan@marmara.edu.tr

E. A. Sánchez Pérez, *Corresponding author. Instituto Universitario de Matemática

Pura y Aplicada, Universitat Politècnica de València, Camino de Vera s/n, 46022 VALEnCia, SPAin.

E-mail address: easancpe@mat.upv.es 